\title{
Smart Distance Lab: A new methodology for assessing social distancing interventions
}

Tessa F. Blanken ${ }^{1, *}$, Charlotte C. Tanis ${ }^{1}$, Floor H. Nauta ${ }^{1}$, Fabian Dablander ${ }^{1}$, Bonne Zijlstra ${ }^{2}$, Rick R.M. Bouten ${ }^{3}$, Quinten H. Oostvogel ${ }^{3}$, Meier J. Boersma ${ }^{4}$, Maya V. van der Steenhoven ${ }^{4}$, Frenk van Harreveld ${ }^{1,5}$, Sanne de Wit ${ }^{1}$, Denny Borsboom ${ }^{1}$

${ }^{1}$ University of Amsterdam, Department of Psychology, 1018 WT Amsterdam, The Netherlands

${ }^{2}$ University of Amsterdam, Research Institute of Child Development and Education, 1012 WX Amsterdam, The Netherlands

${ }^{3}$ Focus Technologies B.V., 5657 EW Eindhoven, The Neterlands

${ }^{4}$ Smart Distance Lab, 2353 NM Leiderdorp, The Netherlands

${ }^{5}$ National Institute for Public Health and the Environment (RIVM), , 3721 MA Bilthoven, The Netherlands

* Corresponding author: Tessa F. Blanken (t.f.blanken@uva.nl)

\begin{abstract}
In the wake of the COVID-19 pandemic, the central importance of human behaviour in mitigating the spread of the virus has become universally recognized. We present a methodology to systematically assess the effectiveness of behavioural interventions to stimulate social distancing. In addition, we demonstrate the feasibility of this framework in a large-scale natural experiment. In an experimental design, we varied behavioural interventions to evaluate the effect of face masks, walking directions, and immediate feedback on visitors' contacts. We represent visitors as nodes, and their contacts as links in a contact network. Subsequently, we used network modelling to test for differences in these contact networks. We find no evidence that face masks influence social distancing, while unidirectional walking directions and buzzer feedback do positively impact social distancing. The presented methodology represents a practically feasible way to optimize social distancing interventions through scientific research and may directly inform policy.
\end{abstract}




\section{Introduction}

Covid-19 holds the world in its grip. Mid-December 2020, almost a year after the first statement about a new virus reached the WHO on December $31^{\text {st }} 2019$, over 70 million confirmed Covid-19 cases and 1.6 million deaths in 220 countries have been reported (WHO, 2020). The virus continues to spread, and many countries suffer from a second wave of infections. As the world awaits the development, production, and distribution of effective vaccines, we only have one weapon at our disposal in our battle against the Coronavirus: our behaviour.

The World Health Organization (WHO) increasingly and repeatedly stresses the importance of engaging in 'safety behaviours', which include washing hands, wearing face masks, and social distancing. Ultimately, social distancing is the most important of the recommended safety behaviours because, as complex as the virus may be, transmission of the virus requires an infectious person to be in physical proximity to other people. Therefore, in absence of a vaccine, it is pivotal to reduce the number of contacts between people that are in such physical proximity (Haug et al., 2020).

Despite its universally acclaimed importance, it is unclear how we can optimally stimulate social distancing through behavioural interventions. Whereas the effectiveness of policy measures to reduce mobility has been investigated (e.g., Flaxman et al., 2020), the effects of more subtle behavioural interventions largely remains a matter of speculation and debate. One of the most striking examples of this debate concerns the behavioural effects of wearing face masks. Policy makers have expressed concern that face masks could give people a false sense of security, thereby promoting risk taking behaviour leading them to keep less distance (e.g., Lazzarino et al., 2020). In contrast, others argued that face masks remind people to implement safety behaviours resulting in increased compliance with Covid-19 policies (e.g., Betsch et al., 2020). Notably, even after evidence on the medical effects of face masks had accumulated, the debate around policy and implementation persisted due to the uncertainty of the behavioural effects of face mask policies (e.g., Martin et al., 2020).

This debate exemplifies how existing scientific work in psychology and behavioural science can provide support for opposing accounts, confusing and occasionally paralyzing policy. To complicate matters further, Covid-19 challenges our behaviour in ways that have not been anticipated or assessed in previous research. Hence, the relevance of the existing psychological and behavioural science literature to this novel crisis is questionable and cannot readily be translated into policy decisions (Van Bavel et al., 2020; IJzerman et al., 2020). These opposing accounts and the novelty of the Covid-19 pandemic thus warrant only one way forward: to assess the effect of behavioural interventions on social distancing experimentally.

Investigation of the effect of behavioural interventions on social distancing is hampered however, by the lack of suitable methodologies that connect behaviour to relevant epidemiological properties. In the current paper we describe, implement, and evaluate a novel methodological framework suited to directly test the effectiveness of different behavioural interventions on social distancing. The framework is shown in Figure 1 and involves (i) an experimental design; (ii) objective measures of social distancing; and (iii) the representation of people (nodes) and their contacts (links) in a contact network. The proposed methodological framework enables us to evaluate the effectiveness of behavioural interventions on social distancing through network representations (Borsboom et al., 2020). 
We implemented this novel framework in a first experimental study during a three-day art fair in Amsterdam. As shown in Figure 1, in the experimental design we implemented walking direction interventions (bidirectional; unidirectional; no direction) and supplementary interventions (face masks; buzzer feedback when within 1.5 meters of another visitor; no supplementary intervention) in two-hour time slots. See Methods section for details. We objectively measured physical proximity between visitors using a Social Distancing Sensor (SDS) based on ultra-wideband technology. Whenever two visitors came within 1.5 meters from each other, the contact was logged. Subsequently, we used the logged contacts to construct the contact networks of visitors for each experimental condition.

In constructing the networks, we collapsed the contacts over time without losing important information, because only primary infections can be transmitted within a two-hour time frame, and therefore only direct contacts are virologically relevant. In other words, if a visitor is infected during the event, this visitor cannot subsequently infect others due to the incubation period, and as a result the order in which people make contacts can be disregarded. In accordance, here we only focus on the direct paths between nodes in the network, which specify the number of unique contacts between visitors and are encoded in the degree distribution. Finally, we use innovative statistical network methodology, based on an adaptation of Van Duijn (1995) to undirected networks, which allows us to statistically test differences in the degree distribution (i.e., the distribution of the number of contacts) across experimental conditions.

In short, the proposed methodology enables us to assess the effects of interventions directly at the level of behaviour. The methodology is straightforward to implement and provides society with the means to directly evaluate interventions, so that policy can be based on evidence rather than conjecture.

\section{Results}

\section{Descriptives.}

Visitors. Over the course of three days, 787 people visited the art fair, of whom 639 (81.2\%) wore a Social Distancing Sensor and participated in our study ${ }^{1}$, see Table 1. For 392 (61.3\%) visitors, demographic information was available as they completed a questionnaire upon purchasing their ticket. These visitors were, on average $\pm \mathrm{SD}, 42 \pm 16$ years old (14-89 years); 225 (57.3\%) were female; and 335 (85.5\%) of them completed a higher education.

\footnotetext{
${ }^{1}$ On Saturday and Sunday, the art fair opened at 11:00 before the experiment started at 13:30. 74 and 132 visitors, respectively, had entered the art fair between 11:00-13:30, of whom some had already left before the experimental condition started. Therefore, some of the 787 visitors did not participate in our studies, and thus the actual percentage of visitors that wore the SDS is higher than the reported $81 \%$.
} 


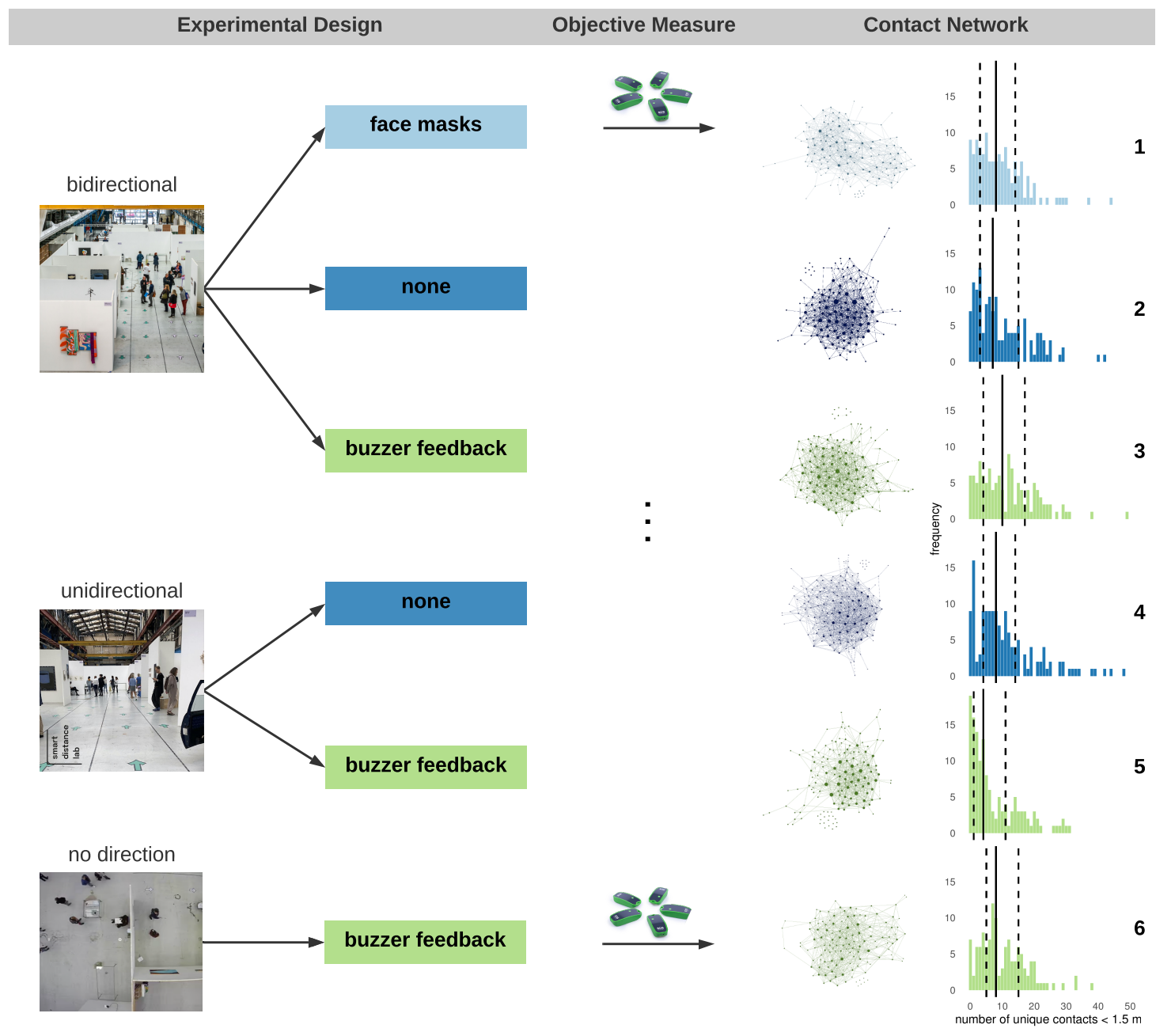

Figure 1. Representation and application of the novel methodological framework. The framework consists of (i) an experimental design; (ii) objective physical distance measures; (iii) a contact network representation. Here, we show the framework applied at the art fair, where we (i) varied walking directions and supplementary interventions; (ii) measured physical distance using a Social Distancing Sensor with ultra-wideband technology; (iii) constructed networks for each condition.

\section{Interventions.}

From the conditions shown in Table 1, we only selected the six conditions that lasted two hours (visualized in Figure 1, see Methods for more elaborate explanation). To evaluate the effectiveness of each behavioural intervention in isolation, (face masks, walking directions, buzzer) we select two conditions that differ only in whether the behavioural intervention of interest is implemented, allowing us to estimate the effect of that intervention.

Face masks. To examine the effect of wearing face masks on social distancing, we compared the contact networks for the face mask condition with the no supplementary intervention condition on the first day 
(condition 1 vs. 2 in Figure 1). As can be seen from Table 1, visitors had a median of 8 unique contacts $(\mathrm{IQR}=3-14$, range $=0-44)$ in the face mask condition, whereas visitors in the no supplementary intervention condition had a median of 7 unique contacts ( $I Q R=3-15$, range $=0-42$ ). Detailed analysis of the probability of visitors forming contacts in these two conditions - taking the network structure of the data into account (see Methods) - did not provide evidence for an effect of wearing face masks on distancing $(O R=1.05,95 \%$ Credible Interval $=[0.81,1.33])$. These results indicate that, if there are differences in the number of contacts when wearing face masks compared to when not wearing them, these differences are likely to be small. In other words, face masks do not appear to facilitate or inhibit social distancing.

Walking direction conditions. We performed two comparisons. First, we evaluated the effect of unidirectional walking directions versus no walking directions by comparing the buzzer conditions on day two and three (condition 5 vs. 6). On day 2 with unidirectional walking directions, visitors had a median of 4 unique contacts (IQR $=1-11$; range $=0-31$ ), whereas on day 3 without walking directions, visitors had a median of 8 unique contacts $(\mathrm{IQR}=5-15$; range $=0-38)$. The probability of visitors forming contacts was substantially higher in the condition with no walking directions compared to the condition with unidirectional walking directions $(O R=1.66,95 \% \mathrm{CI}=[1.25,2.17])$.

Second, we evaluated the effect of bidirectional versus unidirectional walking directions, by comparing the no supplementary intervention conditions on days 1 and 2 (condition 2 vs. 4). During bidirectional walking directions, visitors had a median of 7 unique contacts ( $\mathrm{IQR}=3-15$; range $=0-42$ ), whereas during unidirectional walking directions, visitors had a median of 8 unique contacts (IQR $=4-14$; range $=0-48$ ). The probability of visitors forming contacts was about the same in these two conditions $(O R=0.99,95 \%$ $\mathrm{CI}=[0.75,1.26])$. This indicates that, if there are differences in the number of unique contacts visitors had when given bidirectional versus unidirectional walking directions, these are likely to be small. Thus, the implementation of walking directions appears to improve social distancing, but the differential effects of unidirectional and bidirectional implementations were not statistically significant.

Buzzer. We investigated whether notifying visitors of violations of the $1.5 \mathrm{~m}$ rule through a buzzer reduces the number of contacts by comparing the buzzer condition to the no supplementary intervention condition (condition 2 vs. 3). The median number of unique contacts was $10(\mathrm{IQR}=3-15$; range $=0-42$ ) for visitors in the buzzer condition and $7(\mathrm{IQR}=4-17$; range $=0-49)$ for visitors in the no supplementary intervention condition. The probability of visitors forming contacts was slightly higher in the buzzer condition than in the no supplementary intervention condition $(O R=1.24,95 \% \mathrm{CI}=[0.95,1.55])$. The credible interval around this estimate suggests that buzzers likely do not promote social distancing, and may actually make it worse. In interpreting this effect, it is however important to note that on this day visitors did not receive an instruction on how the buzzer worked, and feedback occurred only two seconds after the contact was made.

After improvements were made to the buzzer intervention (i.e., by implementing a short demonstration and removing the delay between registration of distance violation and feedback) the buzzer did promote distancing (conditions 5 vs. 4). With adequate instructions and immediate feedback, the median number of unique contacts of visitors in the buzzer condition was 4 (IQR $=1-11$; range $=0-31$ ) compared with a median of $8(\mathrm{IQR}=4-14$; range $=0-48)$ unique contacts in the no supplementary condition. The probability of visitors forming contacts was markedly higher in the no supplementary intervention condition, as 
compared to the buzzer conditions $(O R=1.43,95 \% \mathrm{CI}=[1.06,1.91])$. This suggests that buzzer feedback can promote social distancing, but that its effect is dependent on the instructions and immediate feedback.

\section{Discussion}

In this paper we introduced and demonstrated a practical methodology to assess the effect of behavioural interventions on social distancing. The methodology combines systematic variation of interventions in an experimental design with contact network assessment through wearable sensors. Analysing experimentally induced changes in the contact network allows researchers to systematically track the effect of behavioural interventions and as such brings epidemiologically relevant human behaviour within reach of the scientific method. Because it uses assessment of intervention effects at the level of behaviours that are known to affect virus transmission, the introduced methodology can be implemented to bridge the gap between behavioural science and epidemiology. Therefore, this type of research can directly inform policy concerning the mitigation of virus spread.

The implementation of the proposed methodology in the current experimental design may have several policy implications. First, consistent with recent findings from observational research (RosenkrantzLindegaard et al., 2020), we observed no significant effects of wearing face masks on social distancing. This suggests that risk compensation, also known as the Peltzman effect (Prasad \& Jena, 2014), is not operative in the case of face masks. Therefore, even though it is important to replicate the current findings in independent research, the currently available evidence does not support the prominent role that risk compensation has played in deliberations on whether or not to mandate wearing face masks. The finding also underscores the need to execute dedicated empirical research to evaluate behavioural hypotheses, as it illustrates how prima facie plausible claims about human behaviour, backed up by seemingly relevant literature, may miss the mark (IJzerman et al., 2020).

Second, our analysis clearly shows that implementing walking directions lowers the probability of contacts between visitors in the relevant conditions. We hypothesize that this benefit is counteracted by the fact that the movement patterns of other visitors are relatively unpredictable in a free movement scheme, so that distancing requires more effort and attention and accidental collisions occur more frequently. The latter hypothesis could be investigated by subjecting movement patterns isolated from camera data (Tanis et al., 2020) to quantitative pedestrian modelling (Duives, Daamen \& Hoogendoorn, 2013). In comparing different walking directions, we did not observe differential effects of uni- versus bidirectional schemes. Given the dynamics of viral transmission, unidirectional walking directions should be preferred because they mitigate the probability of people facing each other directly; however, in situations where a unidirectional scheme is not available, a bidirectional scheme appears to be a good alternative. The implementation of walking directions is practically feasible in almost all public spaces and the current study directly supports this policy.

Third, our study opens the possibility of utilizing schemes that use sensor data information to provide people with feedback on their behaviour. The current study used a style of feedback most closely related to an alarm: a pattern of light and a buzzer emitted when potential danger is encountered. While this style of feedback does seem to have a positive effect on adherence to distancing guidelines in the current study, we note several challenges with its implementation. First, effective use of sensors seems to depend on 
adequate instruction and immediate feedback. In the absence of this guidance, on the first day of our study, visitors tended to try out the sensors and as such inadvertently came within 1.5 meters of another visitor. This may have obscured the positive effects of feedback on subsequent distancing. After the first day, we countered this effect through a quite elaborate instruction where visitors were invited to try out the sensor on a trial sensor that was not included in the data analysis. However, in many cases such an instruction would be impractical, as it would take time and may lead to queue formation. Second, implementing wearable sensors requires people to pick them up and bring them back, which may generate risky, crowded environments if many people do this at the same time. Currently, the alarm scheme setup warns, but does not reward individuals for keeping distance. Other more rewarding ways to organize feedback may be more engaging (e.g., using gamification by letting people win various awards if they keep their distance) and informative for the user (e.g., many people asked for feedback on how many contacts they made). Clearly, there is a great potential for learning theory (e.g., reinforcement learning) to inform this process (Richter et al., 2015); next to encouraging distancing upon receiving feedback, a feedback system could promote learning to distance effectively and automatically/efficiently.

The present study has yielded experimental support for policy advise concerning physical distancing. However, some limitations warrant attention. First, as we conducted the experiment in a naturalistic setting, we were not in a position to randomize the visitors to time slots. Second, as we were unable to counterbalance all conditions, the design may yield some confounds; for example based on the time of day a condition was measured. Third, as visitors could stay as long as they liked, some visitors were part of multiple conditions creating some dependency in the data. Fourth, as the experiment was conducted at an art fair, the visitors are not a random sample from the population and the results may not generalize to the general population or other settings. At the same time, a naturalistic design has the advantage of high ecological validity. Ultimately, as different contexts draw different crowds and behaviour is affected by one's environment, we should replicate the interventions across various contexts (e.g., in a supermarket, or at a football stadium) to determine which interventions work best in which contexts.

In conclusion, the presented research methodology has a broad range of applications and can be used to assess behavioural interventions quickly and effectively. The current study provides a template for how such research can be organized. Our analysis code (see Appendix) and data (reported separately in Tanis et al., 2020) are available for use in future studies. We hope that this framework will stimulate the behavioural science community to engage with the problem of mitigating virus spread through behavioural interventions.

\section{Methods}

\section{Design.}

Across experimental conditions, we varied two sets of interventions: walking directions and supplementary interventions. Walking directions varied across the three days: bidirectional, unidirectional, and no walking directions respectively, see also Figure 1. Within days, we varied supplementary interventions: face masks, a buzzer that indicated if one came within $1.5 \mathrm{~m}$ of another visitor, no supplementary intervention. While we planned to cross all levels, we deviated from this plan, see "Practical and technological problems". 


\section{Materials.}

Questionnaires. We administered a pre- and post- questionnaire. The pre-questionnaire included items on demographics, potential coronavirus infection, worries about consequences of the virus, social distancing and face masks. The post-questionnaire focussed on social distancing behaviour during the art fair, and the experience of the event. In this paper, we only use visitors' demographics such as age and gender, see (Tanis et al., 2020) for an elaborate description of both questionnaires.

Social Distancing Sensor. In response to the COVID-19 pandemic Focus Technologies B.V. (www.findfocus.nl) together with Sentech B.V. (www.sentech.nl) have developed the Social Distancing Sensor. This sensor is able to determine the distance between itself and other Social Distancing Sensors using ultra-wideband technology with an accuracy of up to ten centimetres. Every sensor has a unique ID and registers how often a violation has occurred with every other sensor. An access point and a computer application are used to manage the system and set settings. Every time a sensor is within reach of the access point, it communicates all distance violations to the access point after which it is written to a central database. A distance violation between two sensors is logged on both sensors and is therefore written to the database twice. The sensor is able to provide direct feedback to the user using a flashing light, a buzzing sensation or a beeping sound. In this research only the light and buzzer are used. To accommodate visitors from the same household, multiple sensors can be paired after which violations of the 1.5 meter distance between these specific sensors are not logged to the database and no feedback is given.

\section{Procedure.}

Via social media, participants were recruited for the event Smart Distance Lab: The Art Fair. Upon purchasing a ticket, visitors were asked to participate in our study by filling out an informed consent form and a questionnaire on demographics and attitudes towards Covid-19. At the end of the questionnaire a code was provided that allowed participants to select a 30 minute time slot to enter the event. If someone declined to participate in our study, they immediately received this code, and participation in our study was thus not required to visit the art fair. There was a separate code that allowed people to receive a free ticket, but the rest of the procedure was the same.

Visitors were allowed to enter the art fair during their selected time slot. Upon entering, it was explained that research was being carried out in the hall and a health check was conducted. At the Social Distance Sensor (SDS) handout station, visitors were asked whether they had signed the informed consent and completed the corresponding questionnaire. Visitors for whom this was not the case were asked whether they wanted to participate in the study and, if so, to sign the informed consent form. When visitors belonged to the same household, this was registered and the sensors were activated at the same time so that they would not report any incidents with each other. In the face mask condition, after handing out the sensors, face masks were handed out with the request to wear them until they were told that they could be removed. Visitors could then enter the art fair and stay inside for as long as they wanted, possibly taking part in multiple experimental conditions. On the way out, visitors handed in their sensors. In addition, they were asked to fill out a post questionnaire about their experience at the art fair.

\section{Practical and technological problems.}


First, the face mask intervention was only executed on day 1, which allows us to only compare the face mask condition to the no supplementary intervention condition on day 1 . Second, on day 3 , two conditions were cut short (60 minutes instead of 120 minutes), see Table 1, and were excluded from analyses. Third, the buzzer notified visitors when they were within $1.5 \mathrm{~m}$ from another visitor, but the settings differed across days. Specifically, on the first day the buzzer only notified people after 2 seconds, which led to a lot of confusion. In addition, we did not actively demonstrate the buzzing feature to the visitors, which resulted in people trying out the feature inside the art fair. On day two and three, visitors were able to test out the buzzer before entering the art fair. In addition, on day two and three the buzzer notified the visitor immediately when they were within $1.5 \mathrm{~m}$ of another visitor, without the two second delay.

\section{Statistical analyses.}

Preprocessing and quality control of sensor data. The SDS output contained the reporting and opposing ID for each contact, the number of times they were within $1.5 \mathrm{~m}$, and the time this contact was registered by the access point. Note that a single contact between two visitors is in this database twice, each visitor once as the 'reporting ID' and once as the 'opposing ID'. First, we separated the output into the different experimental conditions and kept records that were between the start and end of each experimental condition (see Table 1 for start and finish times). Second, for each reporting ID in a condition, we checked whether the sensor was handed out during that condition as registered at the handout station. In case we could not link a sensor to a visitor, we removed this contact from the output. These records could occur when an SDS was activated but not handed out, e.g., when the SDS automatically activated when removed from the charger. Third, as the SDS output only contains records of contacts and the reporting and opposing IDs, we had to add visitors to the output who made zero contacts. More details on the processing of the data can be found in the Open Data paper by Tanis et al. (2020).

Models. The contact networks of visitors were analysed with a logistic regression model including crossnested random effects for the number of contacts of participants. This is a reduced version of the multilevel $\mathrm{p}_{2}$ model (Zijlstra et al., 2006) for non-directed networks, omitting reciprocity parameters and with identical sender and receiver effects. Estimation was performed with Markov Chain Monte Carlo simulations, similar to the estimation of the $\mathrm{j}_{2}$ model (Zijlstra, 2017), with similar prior distributions. In the current application, dummy variables were applied to model differences in the number of contacts between the two networks observed in different conditions. To distinguish this model from others, it was called $\mathrm{b}_{2}$ ( $\mathrm{b}$ for bidirectional). Code for this model is available in the R-package dyads (Zijlstra, 2020).

\section{References}

Bavel JJV, Baicker K, Boggio PS., et al. Using social and behavioural science to support COVID-19 pandemic response. Nat Hum Behav 2020; 4: 460-471. https://doi.org/10.1038/s41562-020-0884-z

Betsch C, Korn L, Sprengholz P, et al. Social and behavioral consequences of mask policies during the COVID-19 pandemic. Proc Natl Acad Sci U.S.A. 2020; 117: 21851-218553.

https://doi.org/10.1073/pnas.2011674117 
Borsboom D, Blanken TF, Dablander F, et al. The lighting of the BECONs: A behavioral data science approach to tracking interventions in COVID-19 research. Preprint at https://doi.org/10.31234/osf.io/53ey9.

Duives DC, Daamen W, Hoogendoorn SP. State-of-the-art crowd motion simulation models. Transp Res Part C Emerg Technol 2013; 37: 193-209. https://doi.org/10.1016/j.trc.2013.02.005

Haug N, Geyrhofer L, Londei A. et al. Ranking the effectiveness of worldwide COVID-19 government interventions. Nat Hum Behav 2020; 4: 1303-1312. https://doi.org/10.1038/s41562-020-01009-0

Hoeben EM, Liebst LS, Bernasco W, et al. Social distancing compliance: A video observational analysis. Preprinted at https://osf.io/59tnu/.

IJzerman H, Lewis NA, Przybylski AK. et al. Use caution when applying behavioural science to policy. Nat Hum Behav 2020; 4: 1092-1094. https://doi.org/10.1038/s41562-020-00990-w

Lazzarino AI, Steptoe A, Hamer M, Michie S. Covid-19: important potential side effects of wearing face masks that we should bear in mind. BMJ 2020; 369: m2003. doi:10.1136/bmj.m2003

Martin GP, Hanna E, McCartney M, Dingwall R. Science, society, and policy in the face of uncertainty: reflections on the debate around face coverings for the public during COVID-19. Crit Public Health 2020; 30: 501-508.

Prasad V, Jena AB. (2014). The Peltzman effect and compensatory markers in medicine. In Healthcare (Vol. 2, No. 3, pp. 170-172). Elsevier.

Richter G, Raban DR, Rafaeli S. (2015). Studying gamification: The effect of rewards and incentives on motivation. In Gamification in education and business (pp. 21-46). Springer, Cham.

Tanis CC, Leach NM, Geiger SJ, et al. The Smart Distance Lab: A new methodology for assessing social distancing interventions. Preprint at https://doi.org/10.31219/osf.io/86sx 7

Van Duijn MA, Snijders TA, Zijlstra BJ. p2: a random effects model with covariates for directed graphs. Statistica Neerlandica 2004; 58: 234-254.

Van Duijn MA. Estimation of a random effects model for directed graphs. In SSS'95. Symposium Statistische Software 1995; 7: 113-131.

Zijlstra BJ, Van Duijn MA, Snijders TA. The Multilevel $\mathrm{p}_{2}$ Model A random effects model for the analysis of multiple social networks. Methodology 2006; 2: 42.

Zijlstra BJ. Regression of directed graphs on independent effects for density and reciprocity. $J$ Math Sociol 2017; 41: 185-192.

Bonne J.H. Zijlstra (2020). dyads: Dyadic Network Analysis. R package version 1.1.3. https://CRAN.Rproject.org/package $=$ dyads

WHO. Coronavirus disease (COVID-19) pandemic. https://www.who.int/emergencies/diseases/novelcoronavirus-2019 (retrieved December 16th) 


\section{Author contributions.}

TFB, CCT, FvH, SdW, and DB developed the study design and behavioural interventions. TFB, CCT, FHN, and DB collected the data. FHN processed the SDS and questionnaire data, and ran the statistical analyses under supervision of TFB, CCT, FD and BZ. BZ developed the statistical models. RRMB and QHO provided the SDSs. MJB and MvdS conceived the Smart Distance Lab initiative. TFB, CCT, and DB wrote the manuscript. FvH and SdW developed the questionnaire content. All authors commented on the manuscript, and reviewed and approved the final manuscript

\section{Acknowledgements.}

We thank photographer Matthijs Immink for allowing us to include his photos of the art fair.

\section{Declaration of Interest.}

R.R.M.B. and Q.H.O. are employed by Focus Technologies B.V., the company that develops and provides the Social Distancing Sensor.

\section{Financial Support.}

The research project was supported by the Ministry of Economic Affairs and Climate Policy. TFB and CCT are supported by an Innovation Exchange Amsterdam UvA Proof of Concept Fund. FD is supported by ZonMw project 10430022010001 . 
Table 1.

Descriptives per Condition

\begin{tabular}{|c|c|c|c|c|c|c|c|c|c|c|c|c|}
\hline \multirow{2}{*}{\multicolumn{2}{|c|}{ Condition }} & & \multirow[t]{2}{*}{$\mathrm{n}$} & \multirow{2}{*}{$\begin{array}{l}\text { Durati } \\
\text { on } \\
(\min )\end{array}$} & \multirow{2}{*}{$\begin{array}{l}\text { Pre Q } \\
(\%)\end{array}$} & \multirow{2}{*}{$\begin{array}{l}\text { Post Q } \\
(\%)\end{array}$} & \multirow{2}{*}{$\begin{array}{l}\text { Age } \\
\text { mean } \\
( \pm \mathrm{SD})\end{array}$} & \multirow{2}{*}{$\begin{array}{l}\text { Males : } \\
\text { Females } \\
\mathrm{n}(\%): \\
\mathrm{n}(\%)\end{array}$} & \multicolumn{4}{|c|}{ Number of contacts $<1.5 \mathrm{~m}$} \\
\hline & & & & & & & & & Range & Mean & Median & IQR \\
\hline 1 & $\begin{array}{l}08 / 28 \\
13: 30-15: 30\end{array}$ & $\begin{array}{l}\text { Face mask } \\
\text { bidirectional }\end{array}$ & 130 & 120 & $\begin{array}{l}66 \\
(51 \%)\end{array}$ & $\begin{array}{l}41 \\
(32 \%)\end{array}$ & $\begin{array}{l}41.3 \\
( \pm 17.0)\end{array}$ & $\begin{array}{l}32(48 \%) \\
34(52 \%)\end{array}$ & $0-44$ & $\begin{array}{l}9.3 \\
( \pm 7.8)\end{array}$ & 8 & $3-14$ \\
\hline 2 & $\begin{array}{l}08 / 28 \\
15: 30-17: 30\end{array}$ & $\begin{array}{l}\text { No intervention } \\
\text { bidirectional }\end{array}$ & 137 & 120 & $\begin{array}{l}77 \\
(56 \%)\end{array}$ & $\begin{array}{l}35 \\
(32 \%)\end{array}$ & $\begin{array}{l}43.2 \\
( \pm 15.1)\end{array}$ & $\begin{array}{l}34(44 \%) \\
43(56 \%)\end{array}$ & $0-42$ & $\begin{array}{l}9.8 \\
( \pm 8.5)\end{array}$ & 7 & $3-15$ \\
\hline 3 & $\begin{array}{l}08 / 28 \\
17: 30-19: 30\end{array}$ & $\begin{array}{l}\text { Buzzer } \\
\text { bidirectional }\end{array}$ & 122 & 120 & $\begin{array}{l}66 \\
(54 \%)\end{array}$ & $\begin{array}{l}24 \\
(20 \%)\end{array}$ & $\begin{array}{l}43.9 \\
( \pm 13.6)\end{array}$ & $\begin{array}{l}24(36 \%) \\
42(64 \%)\end{array}$ & $0-49$ & $\begin{array}{l}11.7 \\
( \pm 8.9)\end{array}$ & 10 & $4-17$ \\
\hline 4 & $\begin{array}{l}08 / 29 \\
13: 30-15: 30\end{array}$ & $\begin{array}{l}\text { No intervention } \\
\text { unidirectional }\end{array}$ & 147 & 120 & $\begin{array}{l}86 \\
(59 \%)\end{array}$ & $\begin{array}{l}40 \\
(27 \%)\end{array}$ & $\begin{array}{l}43.4 \\
( \pm 16.0)\end{array}$ & $\begin{array}{l}38(45 \%) \\
46(55 \%)\end{array}$ & $0-48$ & $\begin{array}{l}11.0 \\
( \pm 9.9)\end{array}$ & 8 & $4-14$ \\
\hline 5 & $\begin{array}{l}08 / 29 \\
16: 00-18: 00\end{array}$ & $\begin{array}{l}\text { Buzzer } \\
\text { unidirectional }\end{array}$ & 137 & 120 & $\begin{array}{l}78 \\
(57 \%)\end{array}$ & $\begin{array}{l}32 \\
(23 \%)\end{array}$ & $\begin{array}{l}43.4 \\
( \pm 16.9)\end{array}$ & $\begin{array}{l}31(40 \%) \\
46(60 \%)\end{array}$ & $0-31$ & $\begin{array}{l}7.3 \\
( \pm 7.8)\end{array}$ & 4 & $1-11$ \\
\hline 6 & $\begin{array}{l}08 / 30 \\
13: 30-15: 30\end{array}$ & $\begin{array}{l}\text { Buzzer no } \\
\text { direction }\end{array}$ & 123 & 120 & $\begin{array}{l}62 \\
(50 \%)\end{array}$ & $\begin{array}{l}18 \\
(15 \%)\end{array}$ & $\begin{array}{l}42.2 \\
( \pm 15.7)\end{array}$ & $\begin{array}{l}23(37 \%) \\
39(63 \%)\end{array}$ & $0-38$ & $\begin{array}{l}10.3 \\
( \pm 7.5)\end{array}$ & 8 & $5-15$ \\
\hline $7^{\mathrm{a}}$ & $\begin{array}{l}08 / 30 \\
15: 30-16: 30\end{array}$ & $\begin{array}{l}\text { Buzzer no } \\
\text { direction }\end{array}$ & 146 & 60 & $\begin{array}{l}79 \\
(54 \%)\end{array}$ & $\begin{array}{l}22 \\
(15 \%)\end{array}$ & $\begin{array}{l}42.8 \\
( \pm 16.1)\end{array}$ & $\begin{array}{l}31(39 \%) \\
48(61 \%)\end{array}$ & $0-28$ & $\begin{array}{l}8.2 \\
( \pm 6.1)\end{array}$ & 7 & $4-11$ \\
\hline $8^{\mathrm{a}}$ & $\begin{array}{l}08 / 30 \\
16: 30-17: 30\end{array}$ & $\begin{array}{l}\text { No intervention } \\
\text { no direction }\end{array}$ & 102 & 60 & $\begin{array}{l}51 \\
(50 \%)\end{array}$ & $\begin{array}{l}3 \\
(3 \%)\end{array}$ & $\begin{array}{l}39.8 \\
( \pm 16.0)\end{array}$ & $\begin{array}{l}20(39 \%) \\
31(61 \%)\end{array}$ & $0-30$ & $\begin{array}{l}5.9 \\
( \pm 5.3)\end{array}$ & 5 & $2-8$ \\
\hline
\end{tabular}


Note. Information on age and sex is based only on visitors who completed the pre-questionnaire and provided this information ( $\mathrm{n}=3$ did not wish to disclose their sex). Number of visitors in each condition don't add to $\mathrm{N}=639$, as some people stayed for more than one condition.

${ }^{a}$ Condition lasted only 60 minutes and was excluded from analyses. 